\title{
Green Synthesis of Silver Nanoparticles Using Nigella sativa Seeds and Evaluation of Their Antibacterial Activity
}

\author{
Marvit Osman Widdatallah ${ }^{*}$, Alaa Abdulmoneim Mohamed ${ }^{2}$, Ayat Ahmed Alrasheid ${ }^{3}$, \\ Hiba Abbas Widatallah", Layla Fathi Yassin", Sahar Hussein Eltilib1, \\ Shimaa Abdel Rahman Ahmed ${ }^{4}$
}

\begin{abstract}
${ }^{1}$ Department of Pharmacology, Faculty of Pharmacy, University of Medical Sciences and Technology, Khartoum, Sudan ${ }^{2}$ Department of Clinical Pharmacy, Faculty of Pharmacy, University of Medical Sciences and Technology, Khartoum, Sudan ${ }^{3}$ Department of Pharmacognosy, Faculty of Pharmacy, University of Medical Sciences and Technology, Khartoum, Sudan ${ }^{4}$ Department of Pharmaceutical Chemistry, Faculty of Pharmacy, University of Medical Sciences and Technology, Khartoum, Sudan Email: *marvatjazzar35@gmail.com
\end{abstract}

How to cite this paper: Widdatallah, M.O., Mohamed, A.A., Alrasheid, A.A., Widatallah, H.A., Yassin, L.F., Eltilib, S.H. and Ahmed, S.A.R. (2020) Green Synthesis of Silver Nanoparticles Using Nigella sativa Seeds and Evaluation of Their Antibacterial Activity. Advances in Nanoparticles, 9, 41-48. https://doi.org/10.4236/anp.2020.92003

Received: January 26, 2020

Accepted: March 13, 2020

Published: March 16, 2020

Copyright $\odot 2020$ by author(s) and Scientific Research Publishing Inc. This work is licensed under the Creative Commons Attribution International License (CC BY 4.0).

http://creativecommons.org/licenses/by/4.0/

\begin{abstract}
Synthesis of silver nanoparticles using seeds of Nigella sativa as a capping agent was evaluated in this study. Different concentrations of the aqueous extract of $N$. sativa with silver nitrate solution were exposed to sunlight; as a force for acceleration of the formulation. Then the silver nanoparticles were characterized by UV-Vis, scanning electron microscope (SEM) and X-ray diffraction (XRD) techniques. Antibacterial activity of the nanoparticles was investigated against Staphylococcus aureus and Escherichia coli by the disc diffusion method. The characterization of nanoparticles was detected by the change in color to yellow-brown which indicated the formulation of silver nanoparticles. Irregular shapes within range of nanoscale were detected using SEM and XRD techniques. The finding suggests that silver nanoparticles may be effectively used as antibacterial agent.
\end{abstract}

\section{Keywords}

Silver Nanoparticles, Nigella sativa, UV-Vis, SEM, XRD, Antibacterial Activity

\section{Introduction}

In today's world, nanotechnology is a relatively new field, but its structural nanometer dimensions are not new, and in fact, these materials have much significance. Nanotechnology has the potential to provide novel, paradigm-shifting solutions to medical problems. Nanotechnology, which has been defined as the 
engineering and manufacturing of materials at the atomic and molecular scale, offers exclusive tools for developing safer and more efficient medicines (nanomedicines), and provides several potential advantages in drug formulation and delivery [1]. In pharmaceutical trade, nanotechnology may revolutionize the rules and possibilities of drug discovery and change the landscape of pharmaceutical industries. In medicine, nanotechnology application may be referred to as nanomedicine that explains various intriguing possibilities in the healthcare sector [2] [3]. Different methods are currently used for the preparation of metallic nanoparticles (NPs) including physical, chemical, and biological methods. Mechanical milling and high-energy mechanical milling are effective physical methods for synthesizing NPs. The chemical method usually involves use of chemicals for synthesis of nanoparticles which makes them certainly unsuitable against any application as it contains toxic compounds [4]. There has usually been a demand for sustainable, reliable, green, and eco-friendly approaches to synthesize metallic NPs minimizing or even disposing of the use of poisonous and risky chemical substances. synthesis of nanoparticles by biological method is through microbes like Aspergillus flavus, Phoma exigua and plant sources such as Cynodon Dactylon, Glycyrrhiza glabra, Nigella sativa, etc. [5] [6] [7]. Of all the nanoparticles developed and characterized thus far, silver nanoparticles (AgNPs) assume a significant position owing to their inherent characteristic of acting as an antimicrobial agent even in the solid state. Although, its significance was recognized much earlier, silver was not well exploited except for its use in oriental medicine and in coins [8]. It is estimated that nearly 320 tons of AgNPs are manufactured every year and used in nanomedical imaging, biosensing and food products [9]. There is a continuous increase in the number of multidrug resistant bacterial and viral strains due to mutation, pollution and changing environmental conditions [10]. To circumvent this predicament, scientists are trying to develop drugs for the treatment of such microbial infections. Many metal salts and metal nanoparticles have been found to be effective in inhibiting the growth of many infectious bacteria [11]. A number of investigations had emphasized the antimicrobial effect of nanoparticles synthesized from plants and was found to be effective against UTI causing bacteria usually Klebsiella spp., Escherichia coli and Staphylococcus aureus [12]. The present study was aimed to make an effective approach for synthesizing silver nanoparticles using seeds of Nigella sativa as a reducing agent and to assessing the potential for their use in the treatment of urinary tract infection.

\section{Materials and Methods}

\subsection{Preparation of Aqueous Silver Nitrate}

Silver nitrate solution (1 M) was prepared and stored in an amber colored bottle.

\subsection{Preparation of Extract from Seeds of Nigella sativa}

The seeds of Nigella sativa were washed several times with deionized water. The 
extract used for synthesis of silver nanoparticles was prepared by adding $20 \mathrm{~g}$ to $100 \mathrm{~mL}$ of distilled water. The suspension was homogenized and centrifuged, after that the supernatant was collected and the extract obtained was filtered through Whatman No. 1 filter paper, finally, the filtrate was stored at $4^{\circ} \mathrm{C}$ [13].

\subsection{Synthesis of Silver Nanoparticles}

Five different aliquots of the seed extracts $(1-5 \mathrm{~mL})$ were taken separately and $10 \mathrm{~mL}$ of $1 \mathrm{mM}$ silver nitrate solution was added with constant stirring and exposed to sunlight radiation. The colour change of the solution was checked periodically; from yellow to dark brown indicated that the silver nanoparticles were synthesized [13].

\subsection{Characterization of Silver Nanoparticles}

\subsubsection{UV-Vis Spectroscopy Analysis}

The reduction of pure $\mathrm{Ag}^{2+}$ ions was monitored by measuring the UV-Vis spectrum of the silver nanoparticles solution after diluting a small aliquot of the sample in distilled water. UV visible spectroscopy was carried out on UV-1800 (Shimadzu) [13].

\subsubsection{SEM Analysis}

This technique was done using Scanning electron Microscope (SEM) (TESCAN MIRA). Thin films of samples were prepared on a carbon coated copper grid by dropping a very small amount of sample on the grid [13].

\subsubsection{XRD Analysis}

The silver nanoparticle solution was centrifuged at $2500 \mathrm{rpm}$ for $20 \mathrm{~min}$. The pellet was washed three times with $20 \mathrm{~mL}$ of deionized water. The dried powder of silver nanoparticles was collected for the determination of formation of silver nanoparticles (XRD-7000s/7000L Shimedazu) [13].

\subsection{Antimicrobial Activity by Disc Diffusion Method}

The prepared Mueller Hinton agar was poured on to sterile Petri-dishes plates and cultures of $E$. coli and $S$. aureus were swabbed on to the agar plates. Meanwhile, the sterile discs were impregnated with the silver nanoparticle solution and a positive control drug (Ciprofloxacin). The plates were incubated overnight at room temperature then the zone of inhibition was measured [14].

\section{Results and Discussion}

\subsection{Synthesis of Silver Nanoparticles}

Different optimization studies were carried out for the synthesis of silver nanoparticles from Nigella sativa seeds extract, results showed in Figure 1 [14]. Among them, sunlight radiation method and homogenized extract showed better production of silver nanoparticles. The reaction medium confirmed the presence of the silver nanoparticles. The color of the reaction medium gradually changed to 
dark brown because of the surface Plasmon resonance. The surface plasmon resonance (SPR) band is influenced by size, shape, morphology, composition and dielectric environment of the prepared nanoparticles [15].

\subsection{Characterization of Silver Nanoparticles}

\subsubsection{UV-Vis Spectral Analysis}

UV-Vis spectra were recorded for the $N$. sativa silver nanoparticles at different concentrations of silver nitrate solution, the result was showed in Figure 2. As the level of the absorption was found to be increase which indicate more synthesis of silver nanoparticles as was found in previous studies [16]. The visible range of UV spectra for silver ranging 400 to $600 \mathrm{~nm}$, while in is this study the absorption ranged between 450 to $480 \mathrm{~nm}$ [17].

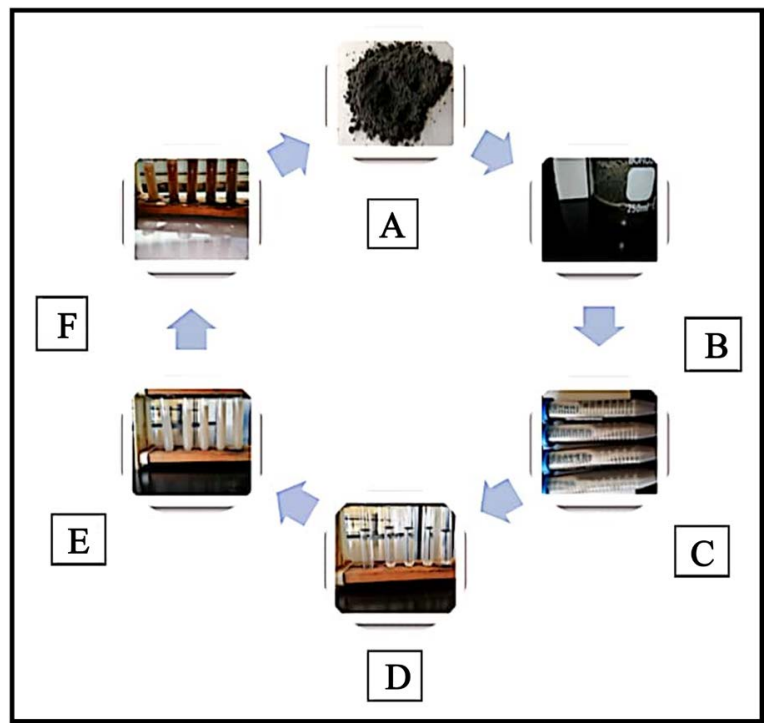

Figure 1. Biosynthesis of Silver nanoparticles. (A) Powder of N. sativa seeds; (B) homogenized suspension; (C) The extract after centrifugation; (D) Silver nitrate $1 \mathrm{M}(10 \mathrm{ml}) ;(\mathrm{E})$ \& (F) The process of reduction after addition of the extract indicated by changing in color.

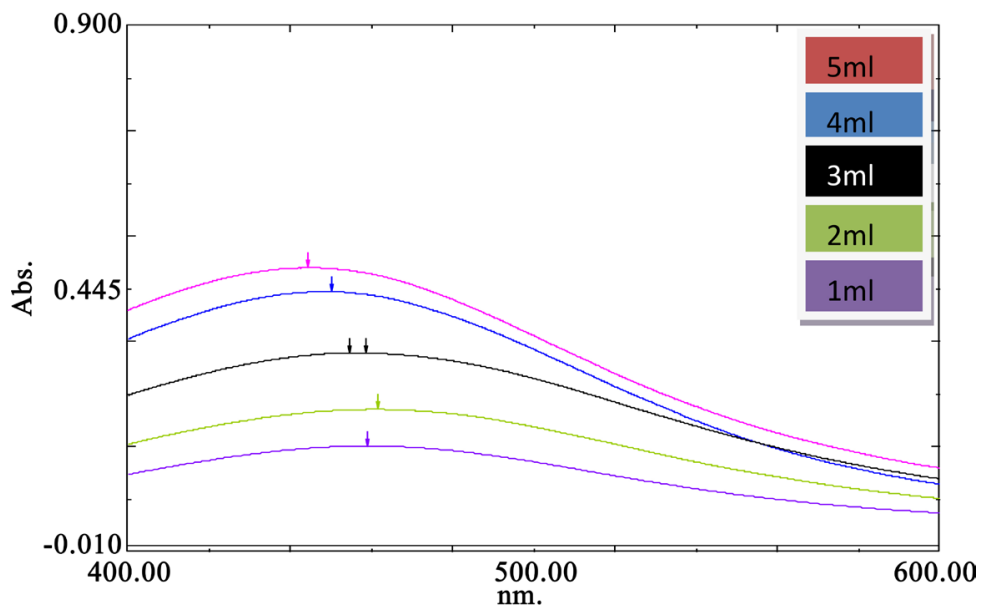

Figure 2. UV-visible spectrum of synthesized silver nanoparticles (AgNPs) using different volumes of the extracts $(1-5 \mathrm{ml})$. 


\subsubsection{SEM Analysis}

Using the Scanning electron microscope technique, a further insight into the morphological details of Nigella sativa AgNPS has been obtained. The SEM micrographs of the synthesized silver nanoparticles showed in Figure 3. The Figure gives information about the irregular morphology and size of the synthesized silver nanoparticles when a voltage of $25 \mathrm{kV}$ was applied. The size was found to be within the actual size range of nanoparticles, which is $1-100 \mathrm{~nm}$ [13].

\subsubsection{XRD Analysis}

The XRD analysis was carried out for size distribution and crystal structure determination. The following results were obtained by the XRD analysis (Figure 4).
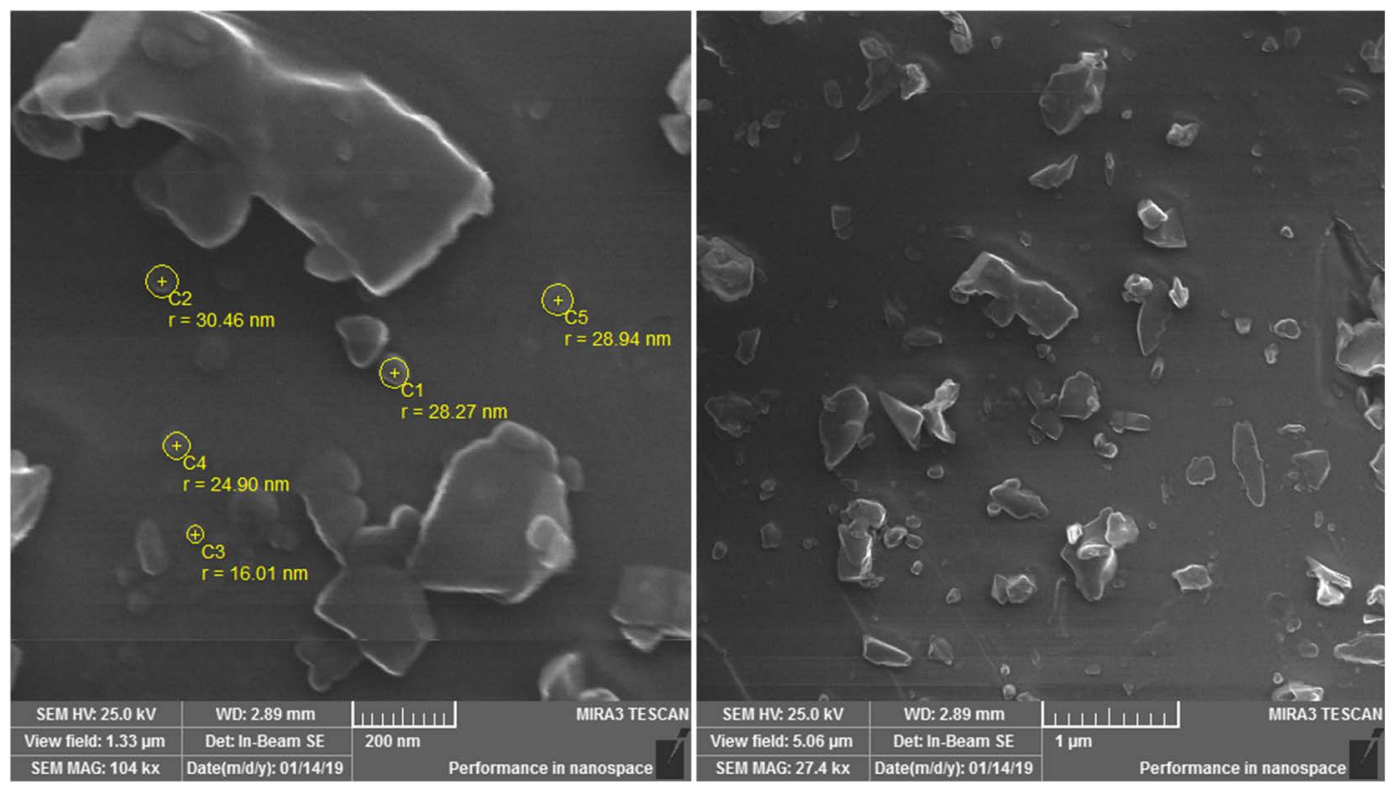

Figure 3. SEM analysis of silver nanoparticles synthesized from Nigella sativa.

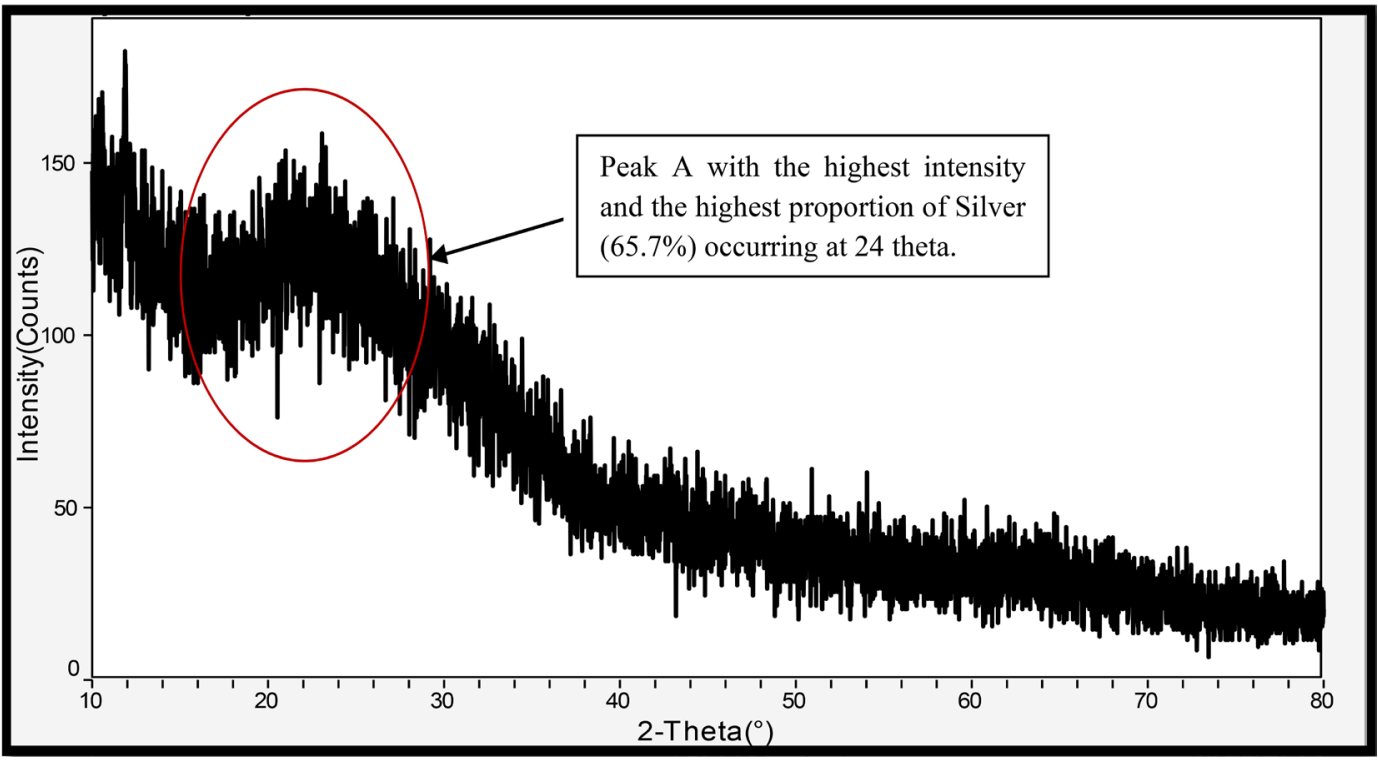

Figure 4. XRD analysis of AgNP synthesized from Nigella sativa. 
Table 1. Antibacterial activity of silver nanoparticles of $N$. sativa seed extract.

\begin{tabular}{ccc}
\hline \multirow{2}{*}{ Sample } & \multicolumn{2}{c}{ Diameter of inhibition zone $(\mathrm{mm})$} \\
\cline { 2 - 3 } & Staphylococcus aureus & Escherichia coli \\
\hline N. sativa $(20 \mathrm{mg} / \mathrm{ml})$ & 13 & 6 \\
Ciprofloxacin & 32 & 18 \\
\hline
\end{tabular}

The synthesized nanoparticles revealed the presence of an orthorhombic shape with three characteristic peaks: Peak A $\left[\mathrm{Ag}_{2}\left(\mathrm{~A}_{14} \mathrm{C}_{110} \mathrm{O}_{2}\right)\right]$, Peak B $\left[\left(\mathrm{AgN}_{3}\right)\left(\mathrm{Ag}\left(\mathrm{NO}_{3}\right)\right)_{2}\right]$ and Peak C [Bis [triamminesilver(I)] bis[diamminesilver(I)] hexafluoridostannate(IV) difluoride $\mathrm{Ag}_{4} \mathrm{~F}_{8} \mathrm{H}_{30} \mathrm{~N}_{10} \mathrm{Sn}$ ]. Peak $\mathrm{A}$ was found to be the most abundant and intense about (65.7\%). In addition, the silver nanoparticles size distribution was estimated using Scherrer Formula found to fall in the range of $(16.20 \mathrm{~nm}$ to $46.06 \mathrm{~nm})$.

\subsection{Antimicrobial Activity}

The antimicrobial activity was tested against two bacterial strains; Gram positive Staphlococcus aureus and Gram negative Escherichia coli. The synthesized AgNPs showed activity against $S$.aureus with inhibition zone of $13 \mathrm{~mm}$ obtained from the highest concentration $(20 \mathrm{mg} / \mathrm{ml})$ while the activity of AgNPs were found to be $6 \mathrm{~mm}$ against $E$. coli. Ciprofloxacin was used in this study as a positive control antibiotic; it obtained higher activity against $S$.aureus compared to E. coli (32 $\mathrm{mm}$ and $18 \mathrm{~mm}$ ) respectively, which is mentioned in Table 1. Thus, the silver nanoparticles of $N$. sativa seeds significantly inhibits the pathogens, however, further investigation is required for understanding the mechanism of action [18].

\section{Conclusion}

In conclusion, the bioreduction of silver ions using seeds of Nigella sativa as reducing agent has been illustrated. From the present study, it is clear that the silver nanoparticles synthesized through the green method using seeds of $N$. sativa can inhibit the organisms causing urinary tract infection providing a significant inhibition zone. Thus, the silver nanoparticles from seeds of $N$. sativa may be used in the management of bacterial urinary tract infections.

\section{Conflicts of Interest}

The authors declare no conflicts of interest regarding the publication of this paper.

\section{References}

[1] Rogers, B., Adams, J. and Pennathur, S. (2014) Nanotechnology: Understanding Small Systems. CRC Press, Boca Raton.

[2] Woodson, T.S. (2016) Public Private Partnerships and Emerging Technologies: A Look at Nanomedicine for Diseases of Poverty. Research Policy, 45, 1410-1418. https://doi.org/10.1016/j.respol.2016.04.005 
[3] Bawa, R. (2016) What's in a Name? Defining "Nano" in the Context of Drug Delivery. In: Handbook of Clinical Nanomedicine: Nanoparticles, Imaging, Therapy, and Clinical Applications, Pan Stanford Publishing, Singapore, 127-168.

https://doi.org/10.1201/b19915

[4] Harstad, S., Hunagund, S., Boekelheide, Z., Hussein, Z.A., El-Gendy, A.A. and Hadimani, R.L. (2018) Gd-Based Magnetic Nanoparticles for Biomedical Applications. In: Magnetic Nanostructured Materials, Elsevier, Amsterdam, 137-155. https://doi.org/10.1016/B978-0-12-813904-2.00005-X

[5] Veisi, H., Pirhayati, M., Kakanejadifard, A., Mohammadi, P., Abdi, M.R., Gholami, J. and Hemmati, S. (2018) In Situ Green Synthesis of Pd Nanoparticles on Tannic Acid-Modified Magnetite Nanoparticles as a Green Reductant and Stabilizer Agent: Its Application as a Recyclable Nanocatalyst (Fe3O4@ TA/Pd) for Reduction of 4-Nitrophenol and Suzuki Reactions. Chemistry Select, 3, 1820-1826.

https://doi.org/10.1002/slct.201702869

[6] Zhu, X., Pathakoti, K. and Hwang, H.M. (2019) Green Synthesis of Titanium Dioxide and Zinc Oxide Nanoparticles and Their Usage for Antimicrobial Applications and Environmental Remediation. In: Green Synthesis, Characterization and Applications of Nanoparticles, Elsevier, Amsterdam, 223-263. https://doi.org/10.1016/B978-0-08-102579-6.00010-1

[7] Singh, J., Dutta, T., Kim, K.H., Rawat, M., Samddar, P. and Kumar, P. (2018) "Green" Synthesis of Metals and Their Oxide Nanoparticles: Applications for Environmental Remediation. Journal of Nanobiotechnology, 16, 84.

https://doi.org/10.1186/s12951-018-0408-4

[8] Siddiqi, K.S., Husen, A. and Rao, R.A. (2018) A Review on Biosynthesis of Silver Nanoparticles and Their Biocidal Properties. Journal of Nanobiotechnology, 16, 14. https://doi.org/10.1186/s12951-018-0334-5

[9] McGillicuddy, E., Murray, I., Kavanagh, S., Morrison, L., Fogarty, A., Cormican, M., Morris, D., et al. (2017) Silver Nanoparticles in the Environment: Sources, Detection and Ecotoxicology. Science of the Total Environment, 575, 231-246. https://doi.org/10.1016/j.scitotenv.2016.10.041

[10] Patel, M.S. (2019) Inactivation of Antibiotic Resistant Bacteria and Genes by Conventional and Advanced Disinfection Methods. Doctoral Dissertation, Rice University, Houston.

[11] Gambino, D. and Otero, L. (2019) Metal Compounds in the Development of Antiparasitic Agents: Rational Design from Basic Chemistry to the Clinic. In: Essential Metals in Medicine: Therapeutic Use and Toxicity of Metal Ions in the Clinic, De Gruyter, Berlin, Boston, 19, 331. https://doi.org/10.1515/9783110527872-013

[12] Mittal, R., Pan, D.R., Parrish, J.M., Huang, E.H., Yang, Y., Patel, A.P., Harjai, K., et al. (2018) Local Drug Delivery in the Urinary Tract: Current Challenges and Opportunities. Journal of Drug Targeting, 26, 658-669. https://doi.org/10.1080/1061186X.2017.1419356

[13] Ranjan, P., Das, M.P., Kumar, M.S., Anbarasi, P., Sindhu, S., Sagadevan, E. and Arumugam, P. (2013) Green Synthesis and Characterization of Silver Nanoparticles from Nigella sativa and Its Application against UTI Causing Bacteria. Journal of Academia and Industrial Research, 2, 45-49.

[14] Safain, K.S. (2017) Screening of Silver Resistance Gene in Clinical Isolates and Determination of Minimum Inhibitory Concentration (MIC) for Silver Nitrate. Doctoral Dissertation, BRAC University, Dhaka. 
[15] Sharma, V., Chotia, C., Ganesan, V. and Okram, G.S. (2017) Influence of Particle Size and Dielectric Environment on the Dispersion Behaviour and Surface Plasmon in Nickel Nanoparticles. Physical Chemistry Chemical Physics, 19, 14096-14106. https://doi.org/10.1039/C7CP01769C

[16] Amooaghaie, R., Saeri, M.R. and Azizi, M. (2015) Synthesis, Characterization and Biocompatibility of Silver Nanoparticles Synthesized from Nigella sativa Leaf Extract in Comparison with Chemical Silver Nanoparticles. Ecotoxicology and Environmental Safety, 120, 400-408. https://doi.org/10.1016/j.ecoenv.2015.06.025

[17] Tafur, G., Benndorf, C., Acosta, D., Asencios, J. and Talledo, A. (2019) Optical Properties of Silver Nanoparticles Embedded in Dielectric Films Produced by dc and rf Magnetron Sputtering. Journal of Physics. Conference Series, 1173, Article ID: 012004. https://doi.org/10.1088/1742-6596/1173/1/012004

[18] Aumeeruddy, M.Z., Zengin, G. and Mahomoodally, M.F. (2018) A Review of the Traditional and Modern Uses of Salvadora persica L. (Miswak): Toothbrush Tree of Prophet Muhammad. Journal of Ethnopharmacology, 213, 409-444.

https://doi.org/10.1016/j.jep.2017.11.030 